\title{
Decentralised Probabilistic Consensus Control for Stochastic Complex Dynamical Networks
}

\author{
Randa Herzallah
}

\begin{abstract}
This paper is concerned with the consensus analysis and control problems for a class of stochastic complex dynamical networks (SCDNs) that consists of a large number of interconnected nodes. In particular, a unified probabilistic decentralised consensus control framework is established where decentralised randomised controllers are designed such that the individual subsystems in a network synchronise their states with each other to achieve consensus of the whole network. The proposed framework is quite general, where all the components within this framework including local controllers, systems' models, and communications between the subsystems of a complex system are modelled using probabilistic models. The general solution for arbitrary probabilistic models of the framework components is obtained then demonstrated on a class of linear Gaussian complex systems, thus obtaining the desired results. Furthermore, a numerical example is presented to illustrate the effectiveness and the usefulness of the theoretical development.
\end{abstract}

Index Terms-Fully Probabilistic Design, coupled stochastic complex systems, consensus control, decentralised control.

\section{INTRODUCTION}

D URING the past years, coordinated consensus has been investigated for multi-agent systems. The goal there is to establish consensus protocols for the design of distributed controllers for a group of dynamic agents such that the network reaches an agreement of certain quantity of interest that depends on the states of all agents. Thereupon, consensus has become a comprehensive interdisciplinary subject where it addresses several research topics such as finite-time consensus for networks of dynamic agents [1], [2] networks with fixed topologies [3] networks with switching topologies [4], formation control [5], [6], and asynchronous consensus [7]. Examples of pioneering results in the field are, the Vicsek model [8], which considered a decentralised control approach and showed that alignment can be achieved through the heading of each agent, and the work by Jadbabaie [9], which provided a detailed mathematical proof of the Vicsek model and presented the jointly connected condition for networks with switching topology.

An important element to designing and analysing consensus control systems is the information exchange between an agent and all of its neighbours in the network. This accessibility to limited and local information by the network agents makes

R. Herzallah is with the Systems Analytics Research Institute, Aston University, Aston Triangle, Birmingham B4 7ET, UK. r.herzallaheaston.ac.uk the design of local controllers, that can achieve network consensus without an intervention of a central controller, very challenging. Therefore, graph theory has been proposed in the literature for characterising the connection between the network subsystems [10]-[12]. In particular, the Laplacian matrix is used in [13] to study the consensus problem of first-order integrator multi agent systems. In addition, the importance of the communication topology has been discussed in [14] and demonstrated on second-order integrator multi agent systems. Since then, the exploitation of graph theory has enabled the transition of the research on consensus to consider other important aspects such as convergence and equilibrium [15]-[18].

The aforementioned results, however, have several disadvantages. They are mostly based on the assumption of linear system dynamics, assume that the dynamics of the agents are known exactly, and assume that the agents are decoupled. These assumptions on the other hand are unrealistic in real world situations. For example, many real-world systems are characterised by nonlinearities and perform badly under linear control. Therefore, recent advances studied and developed the robust analysis for nonlinear networks consensus [19], [20]. These approaches mainly considered consensus control for a class of Lipschitz nonlinear systems. A Kalman filter based nonlinear consensus approach is also developed in [21]. To consider the uncertainty in the evolution of the system dynamics and in signals transmissions and communications, a rich body of the literature has discussed the consensus problem of stochastic complex dynamical networks [22]-[24]. The coupling between the network subsystems, however, has been addressed through synchronisation methods [25]-[27], a topic which is closely related to the consensus of multi-agent systems. For a comprehensive discussion on consensus control, the readers are referred to the surveys [23] and [28] and the references therein.

In spite of that, up until now general results on the design of consensus control for stochastic nonlinear and uncertain complex systems are still lacking. Consequently, this paper will consider the development of a fully probabilistic decentralised control framework for uncertain complex coupled dynamical systems. The main objective of the proposed framework is to design decentralised randomised controllers for the individual subsystems in a complex system such that the divergence between the probabilistic descriptions of neighbouring subsystems is minimised, and at the same time consensus is achieved for the controlled complex system. In particular, the Kullback-Leibler divergence will be employed here to 
characterise the divergence between the probabilistic description of the states of neighbouring subsystems and design the decentralised randomised controllers. The uncertainty of the system dynamics is considered by modelling the stochastic and uncertain dynamical behaviour of the individual subsystems using probabilistic models. To provide coordination between the subsystems' components, the probabilistic message passing proposed in [29] will be adopted here, thus allowing each subsystem to be controlled purely based on local information.

The main contributions of this paper are highlighted as follows: Firstly, a unified probabilistic framework is established where all the components within this framework including local controllers, systems models, and communications between the subsystems of a complex system are modelled using probabilistic models. This probabilistic characterisation of the individual components provides complete descriptions of their behaviours and depicts the inherent uncertainty in their dynamics. Secondly, the proposed framework is more appropriate for application to real world problems, it is for general stochastic, nonlinear and uncertain dynamical systems. Thirdly, the subsystems pass only partial and local information about the states of their dynamics. This partial information is communicated between neighbouring nodes as probabilistic messages and treated as external signals.

The remainder of this paper is organized as follows: Section II formulates the proposed decentralised consensus control problem and presents its general control solution. Section III demonstrates the obtained general solution on linear and Gaussian systems and discusses the probabilistic message passing. These two sections, II and III, include the main results in the paper. To demonstrate the effectiveness of the proposed framework, a numerical example is provided in Section IV. Finally, the conclusion is stated in Section V.

\section{Decentralised RANDomised consensus CONTROL}

This paper will develop pedagogical solutions for decentralised consensus control of stochastic complex dynamical networks (SCDNs), that consist of a large number of interconnected nodes. Here, we consider a complex dynamical network consisting of a collection of $N$ mutually interacting nodes, that are coupled to neighbouring nodes through probabilistic message passing. Within our formulation, the individual nodes are assumed to be described by probabilistic state space models, which can more realistically represent the dynamical behaviour of stochastic systems that are affected by noises and uncertainties. Individual nodes then exchange information with their neighbouring nodes on their states, so that all nodes can work collaboratively to synchronise their behaviours with each other and achieve consensus of the network. Because of the stochastic nature of the nodes constituting a complex dynamical network, the nodes will each be controlled individually by local randomised controllers, $c\left(u_{t ; i} \mid z_{t-1 ; i}\right)$, where $u_{t ; i}$ represents a sequence of multivariate control inputs that is designed to synchronise the dynamics of node $i$ with its neighbours. In particular, the dynamics of the nodes in a SCDN are described by,

$$
\begin{aligned}
& s\left(x_{t ; i}, \tilde{x}_{t ; i}, u_{t ; i} \mid x_{t-1 ; i}, \ldots, x_{0 ; i}, \tilde{x}_{t-1 ; i}, \ldots, \tilde{x}_{0 ; i} u_{t-1 ; i}, \ldots, u_{0 ; i}\right) \\
& =s\left(x_{t ; i} \mid u_{t ; i}, z_{t-1 ; i}\right) s\left(\tilde{x}_{t ; i} \mid \tilde{x}_{t-1 ; i}\right) c\left(u_{t ; i} \mid z_{t-1 ; i}\right)
\end{aligned}
$$

where $z_{t ; i}=\left[x_{t ; i}, \tilde{x}_{t ; i}\right]^{T}$ represents the state vector of subsystem $i$ with $x_{t ; i}$ being the multivariate internal state of subsystem $i$, and $\tilde{x}_{t ; i}$ being the observed external multivariate state received from neighbouring nodes through probabilistic message passing. Also, $s\left(x_{t ; i} \mid u_{t ; i}, z_{t-1 ; i}\right)$, and $c\left(u_{t ; i} \mid z_{t-1 ; i}\right)$ represent the probability density functions (pdfs) of the multivariate state and randomised controller of the local subsystem $i$ respectively, and $s\left(\tilde{x}_{t ; i} \mid \tilde{x}_{t-1 ; i}\right)$ represents the pdf of the external multivariate state to subsystem $i$.

Remark 1: As can be seen from the pdf that characterises the internal state of subsystem $i, s\left(x_{t ; i} \mid u_{t ; i}, z_{t-1 ; i}\right)$, the internal state of subsystem $i$ is in fact coupled to the states of its neighbouring nodes and is affected by their values. On the other hand, the conditioning of the external multivariate state, $s\left(\tilde{x}_{t ; i} \mid \tilde{x}_{t-1 ; i}\right)$ on the previous external state only stems from our assumption that the inherent dynamics of these external variables cannot be influenced by the inputs $u_{t ; i}$, or internal state $x_{t ; i}$ of the $i$ th local subsystem. The assumed form of Equation (1) provides the probabilistic description of stochastic coupled dynamical systems with an example given later in the paper in Equation (12) on linear coupled dynamical systems.

To solve this decentralised consensus control problem such that all subsystems in the network synchronise their behaviours with each other and achieve consensus of the network, the individual randomised controllers need to be derived, such that they meet the constraints imposed by neighbouring subsystems on the states of their corresponding subsystems. Within the fully probabilistic decentralised consensus framework proposed in this paper, these constraints are described through the specification of ideal pdfs from neighbouring subsystems. In particular, the constraint imposed by subsystem $j \in \mathcal{N}_{i}, j \neq i$, is specified by the following ideal pdf,

$$
\begin{aligned}
& { }^{I} s_{j}\left(x_{t ; i}, \tilde{x}_{t ; i}, u_{t ; i} \mid x_{t-1 ; i}, \ldots, x_{0 ; i}, \tilde{x}_{t-1 ; i}, \ldots, \tilde{x}_{0 ; i}\right. \\
& \left., u_{t-1 ; i}, \ldots, u_{0 ; i}\right) \\
& ={ }^{I} s_{j}\left(x_{t ; i} \mid u_{t ; i}, z_{t-1 ; i}\right) s_{j}\left(\tilde{x}_{t ; i} \mid \tilde{x}_{t-1 ; i}\right){ }^{I} c\left(u_{t ; i} \mid z_{t-1 ; i}\right),
\end{aligned}
$$

where here the superscript $I$ is used to denote the ideal pdf of the corresponding factors of pdfs in Equation (1), and $\mathcal{N}_{i}$ is the neighbour set of node $i$ with cardinality $\left|\mathcal{N}_{i}\right|$. Also, note that since $\tilde{x}_{t ; i}$ enters subsystem $i$ as an external signal, its corresponding ideal pdf is taken to be equal to its actual pdf given in Equation (1). This emphasises that the values and pdf of this external signal should not be influenced by the optimised controller of node $i$.

To achieve the consensus of the network objective, randomised controllers need to be derived such that all subsystems in the network synchronise their behaviours with each other. Consequently, the randomised controller of subsystem $i$ is derived such that it minimises the discrepancy between the probabilistic description of the dynamics of its subsystem as given by Equation (1) and the probabilistic description of the dynamics of neighbouring subsystems imposed as ideal distributions, as stated in Equation (2), thus synchronising 
the behaviour of its subsystem with neighbouring subsystems. This discrepancy is measured by the Kullback-Leibler divergence (KLD) as shown in Equation (3) below. Intuitively, if the probabilistic description of the dynamics of subsystem $i$ and the dynamics of its neighbours perfectly match, the value of $-\ln \left(\gamma\left(z_{t-1 ; i}\right)\right)$ will be zero. The lower the $-\ln \left(\gamma\left(z_{t-1 ; i}\right)\right)$ value, the better.

$$
\begin{aligned}
& -\ln \left(\gamma\left(z_{t-1 ; i}\right)\right)=\min _{c\left(u_{t ; i} \mid z_{t-1 ; i}\right)} \sum_{\tau=t}^{T} \int f\left(\mathcal{Z}_{t ; i} \mid \mathcal{Z}_{t-1 ; i}\right) \\
& \times \ln \left(\prod_{j \in \mathcal{N}_{i}, j \neq i} \frac{s\left(x_{t ; i} \mid u_{t ; i}, z_{t-1 ; i}\right) c\left(u_{t ; i} \mid z_{t-1 ; i}\right)}{\left.I_{s_{j}\left(x_{t ; i} \mid u_{t ; i}, z_{t ; i}\right)^{I} c\left(u_{t ; i} \mid z_{t-1 ; i}\right)}\right)}\right) \mathrm{d} \mathcal{Z}_{t ; i} \\
& =\min _{c\left(u_{t ; i} \mid z_{t-1 ; i}\right)} \sum_{\tau=t}^{T} \sum_{j \in \mathcal{N}_{i}, j \neq i} \int f\left(\mathcal{Z}_{t-1 ; i}\right) \\
& \times \ln \left(\frac{s\left(x_{t ; i} \mid u_{t ; i}, z_{t-1 ; i}\right) c\left(u_{t ; i} \mid z_{t-1 ; i}\right)}{I_{s_{j}}\left(x_{t ; i} \mid u_{t ; i}, z_{t-1 ; i}\right)^{I} c\left(u_{t ; i} \mid z_{t-1 ; i}\right)}\right) \mathrm{d} \mathcal{Z}_{t ; i}
\end{aligned}
$$

where $-\ln \left(\gamma\left(z_{t-1 ; i}\right)\right)$ is the expected minimum cost-to-go function, $f\left(\mathcal{Z}_{t ; i}\right)=\prod_{t=1}^{T} s\left(x_{t ; i} \mid u_{t ; i}, z_{t-1 ; i}\right) s\left(\tilde{x}_{t ; i} \mid \tilde{x}_{t-1 ; i}\right) c\left(u_{t ; i} \mid z_{t-1 ; i}\right)$ is the joint distribution of the closed loop dynamic of node $i, \mathcal{Z}_{t ; i}=$ $\left\{z_{t ; i}, \ldots, z_{T ; i}, u_{t ; i}, \ldots, u_{T ; i}\right\}$ is the closed loop observed data sequence, and $T \leq \infty$ is a given control horizon. In addition, note that since the ideal distribution of the external state as specified in Equation (2) is taken to be equal to their corresponding actual pdf given in Equation (1), the pdf of the external state, $s\left(\tilde{x}_{t ; i} \mid \tilde{x}_{t-1 ; i}\right)$ has disappeared from the argument of the ln function in Equation (3). This is due to our realistic assumption that the values and pdfs of these external signals should not be influenced by the optimised controller of node $i$.

Following the same procedure of the conventional centralised FPD [30], using this new definition of the expected cost-to-go function as given in Equation (3), minimisation is then performed recursively to give the following recurrence functional equation,

$$
\begin{aligned}
& -\ln \left(\gamma\left(z_{t-1 ; i}\right)\right)=\sum_{j \in \mathcal{N}_{i}, j \neq i} \int s\left(x_{t ; i} \mid u_{t ; i}, z_{t-1 ; i}\right) s\left(\tilde{x}_{t ; i} \mid \tilde{x}_{t-1 ; i}\right) \\
& \times c\left(u_{t ; i} \mid z_{t-1 ; i}\right)\left[\ln \left(\frac{s\left(x_{t ; i} \mid u_{t ; i}, z_{t-1 ; i}\right) c\left(u_{t ; i} \mid z_{t-1 ; i}\right)}{I_{s_{j}}\left(x_{t ; i} \mid u_{t ; i}, z_{t-1 ; i}\right)^{I} c\left(u_{t ; i} \mid z_{t-1 ; i}\right)}\right)\right. \\
& \left.-\gamma\left(z_{t ; i}\right)\right] \mathrm{d} x_{t ; i} \mathrm{~d} \tilde{x}_{t ; i} \mathrm{~d} u_{t ; i} .
\end{aligned}
$$

The above equation constitutes the recurrence equation of the dynamic programming solution to the consensus decentralised control problem. Its derivation can be obtained following the same procedure discussed in [30].

\section{A. General solution to the randomised decentralised consensus control}

Following the above representations, the general solution for the optimal randomised controller as can be obtained from the recurrence functional equation defined in Equation (4), is given in the following proposition.

Proposition 1: The optimal randomised controller that can be obtained from the recurrence functional equation defined in Equation (4), subject to the joint distribution of the stochastic system given in Equation (1), and its ideal distribution given in Equation (2), is given by,

$c\left(u_{t ; i} \mid z_{t-1}\right)=\frac{{ }_{c} c\left(u_{t ; i} \mid z_{t-1 ; i}\right) \exp \left[-\frac{1}{\left|\mathcal{N}_{i}\right|} \sum_{j \in \mathcal{N}_{i}, j \neq i} \beta_{j}\left(u_{t ; i}, z_{t-1 ; i}\right)\right]}{\gamma\left(z_{t-1 ; i}\right)}$,

$$
\begin{aligned}
& \gamma\left(z_{t-1 ; i}\right)=\int I_{c}\left(u_{t ; i} \mid z_{t-1 ; i}\right) \\
& \times \exp \left[-\frac{1}{\left|\mathcal{N}_{i}\right|} \sum_{j \in \mathcal{N}_{i}, j \neq i} \beta_{j}\left(u_{t ; i}, z_{t-1 ; i}\right)\right] \mathrm{d} u_{t ; i} \\
& \beta_{j}\left(u_{t ; i}, z_{t-1 ; i}\right)=\int s\left(x_{t ; i} \mid u_{t ; i}, z_{t-1 ; i}\right) \\
& \times\left\{\ln \left(\frac{s\left(x_{t ; i} \mid u_{t ; i}, z_{t-1 ; i}\right)}{I_{s_{j}}\left(x_{t ; i} \mid u_{t ; i}, z_{t-1 ; i}\right)}\right)-\ln \tilde{\gamma}\left(x_{t ; i} ; \tilde{x}_{t-1 ; i}\right)\right\} \mathrm{d} x_{t ; i} \\
& \ln \left(\tilde{\gamma}\left(x_{t ; i}, \tilde{x}_{t-1 ; i}\right)\right)=\int s\left(\tilde{x}_{t ; i} \mid x_{t-1 ; i}\right) \ln \left(\gamma\left(z_{t ; i}\right)\right) \mathrm{d} \tilde{x}_{t ; i} .
\end{aligned}
$$

Proof: The derivation of the above result can be reached by evaluating the recurrence equation defined in Equation (4). Using Fubini's theorem, the definitions stated in Equations (7) and (8) can be obtained. Substituting Equations (7) and (8) in Equation (4) yields,

$$
\begin{aligned}
& -\ln \gamma\left(z_{t-1 ; i}\right)=\int \sum_{j \in \mathcal{N}_{i}, j \neq i} c\left(u_{t ; i} \mid z_{t-1 ; i}\right)\left[\beta_{j}\left(u_{t ; i}, z_{t-1 ; i}\right)\right. \\
& \left.+\ln \frac{c\left(u_{t ; i} \mid z_{t-1 ; i}\right)}{{ }^{I} c\left(u_{t ; i} \mid z_{t-1 ; i}\right)}\right] \mathrm{d} u_{t ; i}, \\
& =\int c\left(u_{t ; i} \mid z_{t-1 ; i}\right)\left[\sum_{j \in \mathcal{N}_{i}, j \neq i} \beta_{j}\left(u_{t ; i}, z_{t-1 ; i}\right)\right. \\
& \left.+\left|\mathcal{N}_{i}\right| \ln \frac{c\left(u_{t ; i} \mid z_{t-1 ; i}\right)}{I_{c\left(u_{t ; i} \mid z_{t-1 ; i}\right)}}\right] \mathrm{d} u_{t ; i}, \\
& =\int\left|\mathcal{N}_{i}\right| c\left(u_{t ; i} \mid z_{t-1 ; i}\right)
\end{aligned}
$$

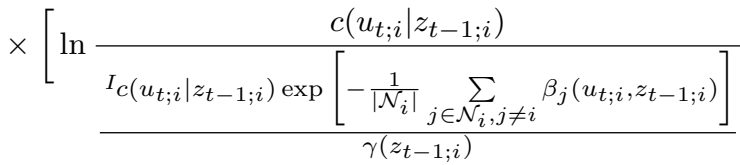

$$
\begin{aligned}
& \left.-\ln \gamma\left(z_{t-1 ; i}\right)\right] \mathrm{d} u_{t ; i} .
\end{aligned}
$$

To clarify, the randomised control solution given in Equation (5) provides the general solution for any arbitrary pdf of the subsystems' dynamics given the constraints imposed by the neighbouring subsystems specified by any arbitrary ideal distributions. However, the evaluation of the analytic solution for this randomised controller is not possible except for the special case of linear and Gaussian pdfs. Therefore, to facilitate the understanding of the proposed decentralised consensus control framework, the solution obtained in Equation (5) will be further demonstrated and obtained for stochastic systems that consist of subsystems, that are characterised by linear and Gaussian distributions.

\section{RANDOMISED CONTROLLERS FOR GAUSSIAN PROBABILISTIC MODELS}

The development in this section will demonstrate the analytic solution for the special case of linear and Gaussian pdfs.

Assume that the stochastic dynamics of subsystem $i$ is given by the following Gaussian conditional pdf,

$$
\begin{aligned}
& s\left(x_{t ; i} \mid z_{t-1 ; i}\right) \sim N\left(\mu_{t ; i}, \Sigma_{i}\right), \\
& s\left(\tilde{x}_{t ; i} \mid \tilde{x}_{t-1 ; i}\right) \sim N\left(\tilde{\mu}_{t ; i}, \tilde{\Sigma}_{i}\right),
\end{aligned}
$$

where,

$$
\begin{aligned}
\mu_{t ; i} & =A_{1 ; i} x_{t-1 ; i}+\sum_{j \in \mathcal{N}_{i}, j \neq i} h l_{i j} x_{t-1 ; j}+B_{i} u_{t ; i} \\
& =A_{1 ; i} x_{t-1 ; i}+A_{2 ; i} \tilde{x}_{t-1 ; i}+B_{i} u_{t ; i} \\
\tilde{\mu}_{t ; i} & =A_{3 ; i} \tilde{x}_{t-1 ; i}
\end{aligned}
$$


and where $\mu_{t ; i}$, and $\tilde{\mu}_{t ; i}$ are the means of the multivariate internal and external states respectively, $l_{i j}$ corresponds to the elements of the Laplacian matrix that specifies the connections between the individual subsystems, and $h$ represents the coupling strength matrix between the connected subsystems. Also, $A_{1 ; i}$ is the internal state matrix, $B_{i}$ is the control input matrix, $\Sigma_{i}$, and $\tilde{\Sigma}_{i}$ are the covariances of the internal and external states respectively. Additionally, the coupling from neighbouring subsystems, $\sum_{j \in \mathcal{N}_{i}, j \neq i} h l_{i j} x_{t-1 ; j}$ in Equation (12) has been rewritten in compact form to explicitly state that the states from neighbouring subsystems, $x_{t-1 ; j}$ enter subsystem $i$ as external states, $\tilde{x}_{t-1 ; i}$. Specifically, $A_{2, i}$ is a matrix whose elements are $h l_{i, j}$ such that $A_{2, i}=\left[h l_{i j}\right]_{j \in \mathcal{N}_{i}, j \neq i}$, and $\tilde{x}_{t-1 ; i}$ is a transpose vector whose elements are the states of neighbouring subsystems, $x_{t-1 ; j}$ such that $\tilde{x}_{t-1 ; i}=\left[x_{t-1 ; j}^{T}\right]_{j \in \mathcal{N}_{i}, j \neq i}^{T}$.

As discussed before, to achieve the control objective of the considered decentralised consensus problem, all subsystems have to synchronise their behaviours with each other. Therefore, each subsystem in the network imposes constraints on its neighbours through the specification of an ideal distribution. Because the objective is for neighbouring subsystems to synchronise their behaviours or states, the ideal distribution of subsystem $j$ on subsystem $i$ is specified as,

$$
\begin{aligned}
I_{s_{j}}\left(x_{t ; i} \mid z_{t-1 ; i}\right) & \sim N\left(\mu_{t ; j}, \Sigma_{2}\right), \\
I_{s_{j}}\left(\tilde{x}_{t ; i} \mid \tilde{x}_{t-1 ; i}\right) & \sim N\left(\tilde{\mu}_{t ; i}, \tilde{\Sigma}_{i}\right),
\end{aligned}
$$

where $\Sigma_{2}$ specifies the desired fluctuations of subsystem $i$ around the mean value of the state of subsystem $j$ that subsystem $i$ needs to synchronise with. Also, note that ${ }^{I} s_{j}\left(\tilde{x}_{t ; i} \mid \tilde{x}_{t-1 ; i}\right)$ is taken to be the same as subsystem $i$ distribution of the external signals, emphasising that the external signals should not be governed or even affected by subsystem $i$ 's state. Similarly, the ideal distribution of the controller as specified collectively from all neighbouring subsystems is assumed to be given by,

$$
{ }^{I} c\left(u_{t ; i} \mid z_{t-1 ; i}\right) \sim N\left(\nu_{t ; i}, \Gamma_{i}\right),
$$

where $\Gamma_{i}$ specifies the admissible range of control inputs of subsystem $i$ that are centred around a mean value, $\nu_{t ; i}$, and where both, $\Gamma_{i}$ and $\nu_{t ; i}$, being tuning parameters which need to be tuned to achieve the optimal results. The next theorem specifies the solution to the optimised randomised controller based on (4), for subsystems with observed external signals.

Theorem 1: The optimal randomised controller for the subsystem described by Equations (10) and (11) and ideal distributions of system dynamics and control inputs described by Equations (14)-(16) is given by,

$$
c\left(u_{t ; i} \mid z_{t-1 ; i}\right)=N\left(\bar{u}_{t ; i}, \bar{\Gamma}_{t ; i}\right)
$$

where

$$
\begin{aligned}
& \bar{u}_{t ; i}=-K_{t ; i} x_{t-1 ; i}+L_{t ; i}, \\
& \bar{\Gamma}_{t ; i}=\left(\Gamma_{i}^{-1}+B_{i}^{T} S_{t ; i} B_{i}\right)^{-1}, \\
& K_{t ; i}=\bar{\Gamma}_{t ; i} B_{i}^{T} S_{t ; i} A_{1 ; i}, \\
& S_{t ; i}=\left(\Sigma_{2}^{-1}+M_{1 t ; i}\right), \\
& L_{t ; i}=-\bar{\Gamma}_{t ; i}\left[-\Gamma_{i}^{-1} \nu_{t ; i}+B_{i}^{T} S_{t ; i} A_{2 ; i} \tilde{x}_{t-1 ; i}+0.5 B_{i}^{T} g_{1 t ; i}\right. \\
& \left.-B_{i}^{T} \Sigma_{2}^{-1} \frac{1}{\left|\mathcal{N}_{i}\right|} \sum_{j \in \mathcal{N}_{i}, j \neq i} \mu_{t ; j}+B_{i}^{T} M_{2 t ; i} A_{3 ; i} \tilde{x}_{t-1 ; i}\right],
\end{aligned}
$$

and where,

$$
-\ln \left(\gamma\left(z_{t-1 ; i}\right)\right)=0.5\left[z_{t-1 ; i}^{T} M_{t ; i} z_{t-1 ; i}+g_{t ; i} z_{t-1 ; i}+\omega_{t ; i}\right],
$$

with,

$$
M_{1 t-1 ; i}=A_{1 ; i}^{T} S_{t ; i} A_{1 ; i}-A_{1 ; i}^{T} S_{t ; i} B_{i} \bar{\Gamma}_{t ; i} B_{i}^{T} S_{t ; i} A_{1 ; i},
$$

$$
\begin{aligned}
& M_{2 t-1 ; i}=A_{1 ; i}^{T} S_{t ; i} A_{2 ; i}-A_{1 ; i}^{T} S_{t ; i} B_{i} \bar{\Gamma}_{t ; i} B_{i}^{T} M_{2 t ; i} A_{3 ; i} \\
& +A_{1 ; i}^{T} M_{2 t ; i} A_{3 ; i}-A_{1 ; i}^{T} S_{t ; i} B_{i} \bar{\Gamma}_{t ; i} B_{i}^{T} S_{t ; i} A_{2 ; i}, \\
& M_{3 t-1 ; i}=A_{2 ; i}^{T} S_{t ; i} A_{2 ; i}+2 A_{2 ; i}^{T} M_{2 t ; i} A_{3 ; i}+A_{3 ; i}^{T} M_{3 t ; i} A_{3 ; i} \\
& -\left(A_{2 ; i}^{T} S_{t ; i}+A_{3 ; i}^{T} M_{2 t ; i}\right) B_{i} \bar{\Gamma}_{t ; i} B_{i}^{T}\left(S_{t ; i} A_{2 ; i}+M_{2 t ; i} A_{3 ; i}\right) \text {, } \\
& g_{1 t-1 ; i}=g_{1 t ; i} A_{1 ; i}-2\left[-\Gamma_{i}^{-1} \nu_{t ; i}-B_{i}^{T} \Sigma_{2}^{-1} \frac{1}{\left|\mathcal{N}_{i}\right|} \sum_{j \in \mathcal{N}_{i}, j \neq i} \mu_{t ; j}\right. \\
& \left.+0.5 B_{i}^{T} g_{1 t ; i}\right]^{T} \bar{\Gamma}_{t ; i} B_{i}^{T} S_{t ; i} A_{1 ; i}-\frac{2}{\left|\mathcal{N}_{i}\right|} \sum_{j \in \mathcal{N}_{i}, j \neq i} \mu_{t ; j}^{T} \Sigma_{2}^{-1} A_{1 ; i} \\
& g_{2 t-1 ; i}=g_{2 t ; i} A_{3 ; i}-2\left[-\Gamma_{i}^{-1} \nu_{t ; i}-B_{i}^{T} \Sigma_{2}^{-1} \frac{1}{\left|\mathcal{N}_{i}\right|} \sum_{j \in \mathcal{N}_{i}, j \neq i} \mu_{t ; j}\right. \\
& \left.+0.5 B_{i}^{T} g_{1 t ; i}\right]^{T} \bar{\Gamma}_{t ; i}\left[B_{i}^{T} S_{t ; i} A_{2 ; i}+B_{i}^{T} M_{2 t ; i} A_{3 ; i}\right]+g_{1 t ; i} A_{2 ; i} \\
& -\frac{2}{\left|\mathcal{N}_{i}\right|} \sum_{j \in \mathcal{N}_{i}, j \neq i} \mu_{t ; j}^{T} \Sigma_{2}^{-1} A_{2 ; i} \\
& \omega_{t-1 ; i}=\frac{1}{\left|\mathcal{N}_{i}\right|} \sum_{j \in \mathcal{N}_{i}, j \neq i} \mu_{t ; j}^{T} \Sigma_{2}^{-1} \mu_{t ; j}+\operatorname{tr}\left(M_{1 t ; i} \Sigma_{i}\right) \\
& -\left[-\Gamma_{i}^{-1} \nu_{t ; i}-B_{i}^{T} \Sigma_{2}^{-1} \frac{1}{\left|\mathcal{N}_{i}\right|} \sum_{j \in \mathcal{N}_{i}, j \neq i} \mu_{t ; j}+0.5 B_{i}^{T} g_{1 t ; i}\right]^{T} \\
& \times \bar{\Gamma}_{t ; i}\left[-\Gamma_{i}^{-1} \nu_{t ; i}-B_{i}^{T} \Sigma_{2}^{-1} \frac{1}{\left|\mathcal{N}_{i}\right|} \sum_{j \in \mathcal{N}_{i}, j \neq i} \mu_{t ; j}\right. \\
& \left.+0.5 B_{i}^{T} g_{1 t ; i}\right]+\nu_{t ; i}^{T} \Gamma_{i}^{-1} \nu_{t ; i}+\operatorname{tr}\left(M_{3 t ; i} \tilde{\Sigma}_{i}\right)+\omega_{t ; i} \\
& +\ln \left|I+\left(B_{i} \Gamma_{i}^{0.5}\right)^{T} S_{t ; i}\left(B_{i} \Gamma_{i}^{0.5}\right)\right|,
\end{aligned}
$$

is the quadratic cost function. We have also introduced the following partitioning of the matrices $M_{t ; i}=\left[\begin{array}{cc}M_{1 t ; i} & M_{2 t ; i} \\ M_{2 t ; i}^{T} & M_{3 t ; i}\end{array}\right]$, and $g_{t ; i}=$ $\left[\begin{array}{ll}g_{1 t ; i} & g_{2 t ; i}\end{array}\right]$. In addition, $\bar{u}_{t ; i}$ and $\bar{\Gamma}_{t ; i}$ are the mean and covariance of the optimal randomised controller of subsystem $i$ respectively, $K_{t ; i}$ is the controller feedback gain, and $L_{t ; i}$ is a linear shift which manifests from the considered consensus problem. Furthermore, $M_{t ; i}$ represents the discrete Riccati equation, $g_{t ; i}$, which also manifests from the considered consensus problem, is referred to in this paper as the consensus equation, and $\omega_{t-1 ; i}$ is some positive constant that does not depend on the system state.

Proof: The proof of this theorem can be obtained following the same procedure in [31]. It can be easily achieved by evaluating the elements given in Equation (5) using the corresponding pdfs specified in Equations (10)-(16).

As can be seen from Equation (17), only the two blocks defined in Equations (20) and (21) of the full Riccati matrix $M_{t ; i}$ need to be solved. The third block defined in (22) of the Riccati equation does not need to be solved. Similarly, only $g_{1 t ; i}$ specified in Equation (23) needs to be solved. This decreases the computational efforts in obtaining the optimal randomised control law compared to the global solution.

\section{A. Probabilistic message passing}

This subsection briefly discusses the probabilistic message passing methodology. For more details and the general results of the probabilistic message passing methodology, the readers are referred to [29]. 
As discussed in previous sections, external signals are received as messages from neighbouring nodes to provide coordination between the subsystems and keep them informed about the states of each other. Thus, they play central role in achieving the global consensus control objective of the complex system. To be more specific, consider the complete description of the interacting variables of subsystem $j$, $s\left(x_{t ; j}, \tilde{x}_{t ; i} u_{t ; j} \mid z_{t-1 ; j}\right)$. This subsystem will then send a message to its neighbouring subsystem $i$ specifying the probabilistic description of its own internal state, $x_{t ; j}$. This probabilistic message from subsystem $j$ to $i$ is obtained as follows,

$$
\begin{aligned}
& M_{i \leftarrow j}=\int s\left(x_{t ; j}, \tilde{x}_{t ; j} u_{t ; j} \mid z_{t-1 ; j}\right) \mathrm{d} \tilde{x}_{t ; j} \mathrm{~d} u_{t ; j}, \\
& =\int s\left(x_{t ; j} \mid u_{t ; j}, z_{t-1 ; j}\right) s\left(\tilde{x}_{t ; j} \mid \tilde{x}_{t-1 ; j}\right) c\left(u_{t ; j} \mid z_{t-1 ; j}\right) \mathrm{d} \tilde{x}_{t ; j} \mathrm{~d} u_{t ; j}, \\
& =N\left(\mu_{t ; i \leftarrow j}, \Sigma_{i \leftarrow j}\right),
\end{aligned}
$$

where,

$$
\begin{aligned}
\mu_{t ; i \leftarrow j} & =A_{1 ; j} x_{t-1 ; j}+A_{2 ; j} \tilde{x}_{t-1 ; j}+B_{j} \bar{u}_{t ; j}, \\
\Sigma_{i \leftarrow j} & =B_{j} \Gamma_{t ; j} B_{j}^{T}+\Sigma_{j} .
\end{aligned}
$$

Note that we have integrated over all variables of subsystem $j$ except its internal state. This information about the internal state of subsystem $j$ is then passed to subsystem $i$ and fused with the prior information, that subsystem $i$ retains, in the form of external signals about the state of subsystem $j$. To be more specific, Equation (26) and Equation (11) are fused using Bayes' rule by multiplying the two together, yielding,

$$
\begin{aligned}
s\left(\tilde{x}_{t, i, f \text { used }}\right) & =N\left(\mu_{t ; i \leftarrow j}, \Sigma_{i \leftarrow j}\right) N\left(\tilde{\mu}_{t ; i}, \tilde{\Sigma}_{i}\right), \\
& =N\left(\tilde{\mu}_{t ; i ; \text { fused }}, \tilde{\Sigma}_{t ; i ; \text { fused }}\right),
\end{aligned}
$$

where,

$$
\begin{aligned}
\tilde{\mu}_{t ; i ; \text { used }} & =\tilde{\mu}_{t ; i}+K_{t ; i}\left(\mu_{t ; i \leftarrow j}-\tilde{\mu}_{t ; i}\right), \\
\tilde{\Sigma}_{t ; i ; \text { used }} & =\tilde{\Sigma}_{i}-K_{t ; i} \tilde{\Sigma}_{i}
\end{aligned}
$$

and where,

$$
K_{t ; i}=\tilde{\Sigma}_{i}\left(\tilde{\Sigma}_{i}+\Sigma_{i \leftarrow j}\right)^{-1} .
$$

These equations can then be used to update the parameter $A_{3 ; i}$ in Equation (13) based on linear optimisation methods. For more details on this message passing approach please refer to [29].

\section{B. Algorithm of Theorem one}

The implementation of the controller (17) requires the evaluation of the solutions of the Riccati and consensus equations. For infinite control problems, the solutions of these equations become steady state (SS) solutions and the controller feedback gain becomes a constant matrix. We start by finding the SS solution of Equation (20). One way to achieve this is to reverse the direction of time. Using the definition of $S_{t ; i}$ from Equation (18) and reversing the direction of time, Equation (20) is modified to read,

$$
\begin{aligned}
& M_{1 t ; i}=A_{1 ; i}^{T}\left(\Sigma_{2}^{-1}+M_{1 t-1 ; i}\right) A_{1 ; i} \\
& -A_{1 ; i}^{T}\left(\Sigma_{2}^{-1}+M_{1 t-1 ; i}\right) B_{i} \bar{\Gamma}_{t-1 ; i} B_{i}^{T}\left(\Sigma_{2}^{-1}+M_{1 t-1 ; i}\right) A_{1 ; i} .
\end{aligned}
$$

Starting the solution with $M_{10 ; i}=0$, iterate the equation until a SS solution, $M_{1 ; i}$ is obtained. Using the obtained SS solution, $M_{1 ; i}$ in Equations (21) and (23), the same time reversal procedure can be applied to obtain the SS solutions, $M_{2 ; i}$ and $g_{1 ; i}$, of these two equations. The key steps of the proposed algorithm are summarised as pseudocode in Algorithm 1.

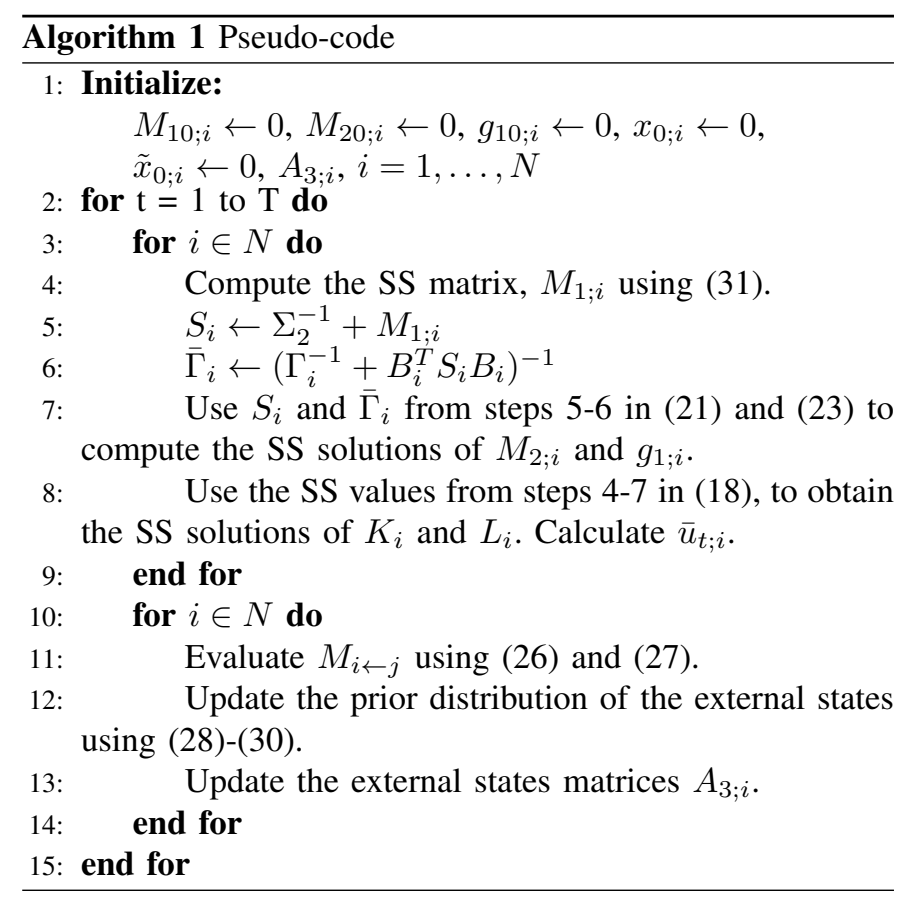

\section{Simulation EXAMPLE}

In order to illustrate the validity of the theoretical development of the proposed probabilistic decentralised consensus control, the theory developed in Section III is applied here to a group of eight stochastic coupled oscillators described by linear and Gaussian pdfs according to Equations (10) and (11) with the following parameters,

$$
\begin{aligned}
\mu_{t ; i} & =A_{1 ; i} x_{t-1 ; i}-\sum_{j \in N_{i}, j \neq i} h l_{i j} x_{t-1 ; j}+B_{i} u_{t ; i}, \\
\tilde{\mu}_{t ; i} & =A_{3 ; i} \tilde{x}_{t-1 ; i}
\end{aligned}
$$

where $A_{1 ; i}=A-h l_{i i}, A=\left[\begin{array}{cc}1 & 0.015 \\ -0.015 & 1\end{array}\right], B_{i}=$ $\left[\begin{array}{ll}0 & 0.015\end{array}\right]^{T}, h=0.03 I_{2 \times 2}, \Sigma_{i}=\Sigma_{2}=0.001 I_{2 \times 2}, I$ is the identity matrix, and where the $l_{i j}$ correspond to the elements of the Laplacian matrix, $L$ which is specified as,

$$
L=\left[\begin{array}{cccccccc}
1 & -1 & 0 & 0 & 0 & 0 & 0 & 0 \\
-1 & 2 & -1 & 0 & 0 & 0 & 0 & 0 \\
0 & -1 & 2 & -1 & 0 & 0 & 0 & 0 \\
0 & 0 & -1 & 2 & -1 & 0 & 0 & 0 \\
0 & 0 & 0 & -1 & 2 & -1 & 0 & 0 \\
0 & 0 & 0 & 0 & -1 & 2 & -1 & 0 \\
0 & 0 & 0 & 0 & 0 & -1 & 2 & -1 \\
0 & 0 & 0 & 0 & 0 & 0 & -1 & 1
\end{array}\right]
$$

Also, note that $A_{3 ; i}$ is initialised randomly at the beginning and then updated according to the probabilistic message passing discussed in Section III-A. A typical example of the network of oscillators of the form defined in Equation (32) is the mass-spring systems [32] with control input being the driving force on the mass.

As stated previously, randomised controllers are designed here such that the discrepancy, as measured by the KLD (3), between the pdf of the state of subsystem $i$ whose parameters are given in Equation (32), and the pdfs of its neighbours is minimised; thus reaching consensus of the whole network. In the current experiment, the initial values of the states of the eight oscillators were set to,

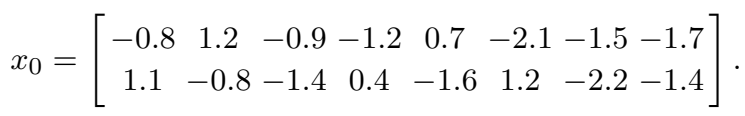

Following Algorithm 1, the states of the system are evaluated and shown in Figures 1, (a) and (b). From these Figures, it can be seen 
that all the states of the eight coupled oscillators are synchronised with each other and that the designed probabilistic controllers have been effective in achieving consensus of the whole network.

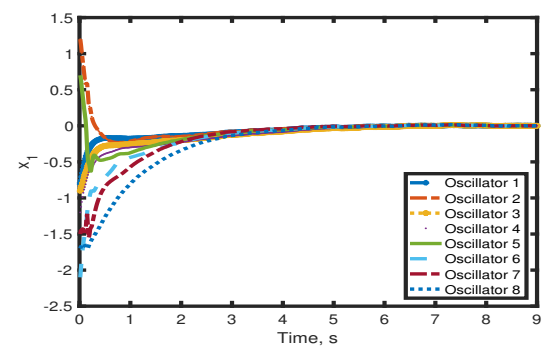

(a)

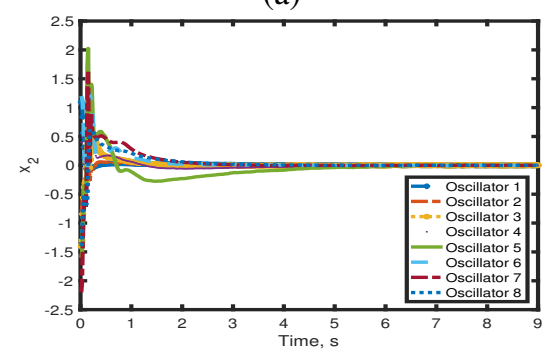

(b)

Fig. 1. The system of eight coupled oscillators. (a) the first state of the eight oscillators. (b) the second state of the eight oscillators.

\section{CONCLUSION}

This paper presented a pedagogic solution for the consensus control of complex stochastic dynamical and coupled systems that consist of a large number of nodes. The obtained solution is quite general, where the stochastic subsystems constituting a complex system are modelled using arbitrary probability density functions. Furthermore, complete information about the states of neighbouring subsystems are passed between the subsystems as probabilistic messages. This consideration of probabilistic models yielded a unified probabilistic framework where randomised controllers are designed, thus accounting for the stochasticity and uncertainty that characterise many real world systems. To facilitate the understanding of the theoretical development, the general solution of the proposed framework is demonstrated on a class of linear and Gaussian dynamical systems and the analytic results are obtained. Finally, a numerical example has been provided to emphasise the effectiveness of theoretical results.

\section{ReFERENCES}

[1] L. Wang and F. Xiao, "Finite-time consensus problems for networks of dynamic agents," IEEE Transactions on Automatic Control, vol. 55, no. 4, pp. 950-955, 2010.

[2] S. Mondal, J. Ghommam, and M. Saad, "Homogeneous finite-time consensus control for higher-order multi-agent systems by full order sliding mode," Journal of Systems Science and Complexity, vol. 31, pp. 1186-1205, 2018.

[3] Y. Shang, "Finite-time consensus for multi-agent systems with fixed topologies. international journal of systems science,' Journal of Systems Science and Complexity, vol. 43, no. 3, pp. 499-506, 2012.

[4] R. Olfati-Saber and R. M. Murray, "Consensus problems in networks of agents with switching topology and time-delays," IEEE Transactions on Automatic Control, vol. 49, no. 9, pp. 1520-1533, 2004.

[5] K.-K. Oh, M.-C. Park, and H.-S. Ahn, "A survey of multi-agent formation control," Automatica, vol. 53, pp. 424-440, 2015.

[6] N. Xuan-Mung and S. K. Hong, "Robust adaptive formation control of quadcopters based on a leaderfollower approach," International Journal of Advanced Robotic Systems, pp. 1-11, 2019.

[7] G. Bracha and S. Toueg, "Asynchronous consensus and broadcast protocols," Journal of the Association for Computing Machinery, vol. 32, no. 4, pp. 824-840, 1985.
[8] T. Vicsek, A. Czirók, E. Ben-Jacob, I. Cohen, and O. Shochet, "Novel type of phase transition in a system of self-driven particles," Physical Review Letters, vol. 75, no. 6, pp. 1226-1229, 1995.

[9] A. Jadbabaie, J. Lin, and A. S. Morse, "Coordination of groups of mobile autonomous agents using nearest neighbor rules," IEEE Transactionson Automatic Control, vol. 48, no. 6, pp. 988-1001, 2003.

[10] Y. Hong, J. Hu, and L. Gao, "Tracking control for multi-agent consensus with an active leader and variable topology," Automatica, vol. 42, pp. $1177-1182,2006$

[11] Z. Li, Z. Duan, G. Chen, and L. Huang, "Consensus of multiagent systems and synchronization of complex networks, a unified viewpoint," IEEE Transactions on Circuits and Systems I, vol. 57, pp. 213-224, 2010.

[12] J. Xiang, W. Wei, and Y. Li, "Synchronized output regulation of linear networked systems," IEEE Transactions on Automatic Control, vol. 54, pp. 1336-1341, 2009.

[13] Olfati-Saber and R. M. Murray, "Consensus problems in networks of agents with switching topology and time-delays," IEEE Transactionson Automatic Control, vol. 49, no. 9, pp. 1520-1533, 2004.

[14] W. Ren and R. W. Beard, "Consensus seeking in multiagent systems under dynamically changing interaction topologies," IEEE Transactionson Automatic Control, vol. 50, no. 5, pp. 665-661, 2005.

[15] M. Zhu and S. Martínez, "Discrete-time dynamic average consensus," Automatica, vol. 46, no. 2, pp. 322-329, 2010.

[16] D. Meng, Y. Jia, J. Du, and J. Zhang, "On iterative learning algorithms for the formation control of nonlinear multiagent systems," Automatica, vol. 50, no. 1, pp. 291-295, 2014.

[17] F. Erkan and M. Akar, "Discrete-time analysis of multi-agent networks with multiple consensus equilibria," IFAC-PapersOnLine, vol. 49, no. 3, pp. 389-394, 2016.

[18] M. M. Gulzar, S. T. H. Rizvi, M. Y. Javed, U. Munir, and H. Asif, "Multiagent cooperative control consensus: A comparative review," Electronics, vol. 7 , no. 22 , pp. 1-20, 2018.

[19] Z. Ding, "Consensus control of a class of lipschitz nonlinear systems," International Journal of Control, vol. 87, no. 11, pp. 2372-2382, 2014.

[20] W. Yu, G. Chen, M. Cao, and J. Kurths, "Second-order consensus for multiagent systems with discrete topologies and nonlinear dynamics," IEEE Transactions on Systems, Man, and Cybernetics, Part B: Cybernetics, vol. 40, pp. 881-891, 2010.

[21] W. Ren, R. W. Beard, and D. B. Kingston, "Multi-agent kalman consensus with relative uncertainty," in American Control Conference, Portland, OR, 2005, pp. 1865-1870.

[22] X. Li, Y. C. Soh, and L. Xie, "Design of output-feedback protocols for robust consensus of uncertain linear multi-agent systems," in American Control Conference, Seattle, USA, 2017, pp. 936-941.

[23] X. Mu and Y. Chen, "A survey of the consensus for multi-agent systems," Systems Science and Control Engineering, vol. 7, no. 1, pp. 468-482, 2019.

[24] H. Ye, M. Li, and W. Luo, "Consensus protocols for heterogeneous multiagent systems with disturbances via integral sliding mode control," Mathematical Problems in Engineering, 2018.

[25] K. H. Movric, K. You, F. L. Lewis, and L. Xie, "Synchronization od discrete-time multi-agent systems on graphs using riccati design," Automatica, vol. 49, no. 2, pp. 414-423, 2013.

[26] L. Wang, M. Z. Q. Chen, and Q.-G. Wang, "Bounded synchronization of a heterogeneous complex switched network," Automatica, vol. 56, pp. $19-24,2015$.

[27] X. Mu and Y. Chen, "Synchronization of delayed discrete-time neural networks subject to saturated time-delay feedback," Neurocomputing, vol. 175, pp. 293-299, 2016.

[28] W. Ren, R. W. Beard, and E. M. Atkins, "A survey of consensus problems in multi-agent coordination," in American Control Conference, Portland, OR, USA, 2005, pp. 1859-1864.

[29] R. Herzallah, "Probabilistic message passing for decentralized control of stochastic complex systems," IEEE Access, vol. 7, pp. $184707-184717$, 2019.

[30] R. Herzallah and M. Kárný, "Fully probabilistic control design in an adaptive critic framework," Neural Networks, vol. 24, no. 10, pp. 11281135, 2011.

[31] R. Herzallah, "Generalised probabilistic control design for uncertain stochastic control systems," Asian Journal of Control, vol. 20, no. 6, pp. 2065-2074, 2018.

[32] Z. Li, Z. Duan, L. Xie, and X. Liu, "Distributed robust control of linear multi-agent systems with parameter uncertainties," International Journal of Control, vol. 85, no. 8, pp. 1039-1050, 2012. 